\title{
Belastungstest mit oder ohne Sauerstoff?
}

\author{
Ein standardisierter körperlicher Belastungstest zur \\ Prognoseabschätzung einer geplanten Lungenteilresektion \\ sollte mit Sauerstoffapplikation erfolgen. Dadurch lassen sich \\ differenziertere Resultate gewinnen. Dies ist das Ergebnis einer \\ Pilotstudie des US-Forscherteams um H. M. Womble.
}

Lung 2012; 190: 263-269

Zur Abschätzung des OP-Risikos erfolgt vor der Lungenteilresektion eine Belastungsergometrie. Entscheidend ist die Sauerstoffaufnahme $\left(\mathrm{VO}_{2} \mathrm{max}\right)$. Bei Werten $<10 \mathrm{ml} / \mathrm{kg} / \mathrm{min}$ gilt der Eingriff als nicht indiziert. Die Autoren vermuten, dass dieses Kriterium auch von der individuellen Reaktion auf eine Sauerstoffgabe während der Ergometrie abhängig gemacht werden sollte. Nach Meinung der Forscher führt die bisherige Testpraxis (Ergometrie ohne zusätzlichen Sauerstoff) dazu, dass bei einigen Betroffenen die OP-Prognose zu ungünstig eingeschätzt wird.

Im Rahmen einer randomisierten, doppelt verblindeten Crossover-Studie wurde der Effekt einer Sauerstoffapplikation bei 16 COPD-Patienten geprüft, bei denen eine Lungenresektion vorgenommen werden sollte. Die Forscher verglichen jeweils eine Testphase bei Raumluft mit der unter Sauerstoffgabe per Nasenbrille (3 l/min). Zielparameter war der Sauerstoffverbrauch während des Belastungstests. Außerdem wurden Standardparameter der körperlichen Belastung, wie z. B. die maximal erreichte Wattzahl, erfasst.

\section{Erhöhte Sauerstoffaufnahme $\nabla$}

Durch die Sauerstoffgabe während des Belastungstests konnte die Sauerstoffaufnahme im Durchschnitt um $11 \%$ gesteigert werden. 4 der 16 Patienten unterschritten ohne Sauerstoffgabe den Schwellenwert. Mit Sauerstoff überschritten 3 der 4 den Schwellenwert $\left(\mathrm{VO}_{2} \max <10 \mathrm{ml} / \mathrm{kg} / \mathrm{min}\right)$ wieder und erwiesen sich so als potenziell operabel. Die maximale Sättigung stiegt durch die Maßnahme an (93 vs. 98\%). Das endtidale $\mathrm{CO}_{2}$ erhöhte sich ebenfalls (37 vs. $40 \mathrm{mmHg}$ ). Auf die physischen Parameter wie Übungszeit (6min), maximale Leistung (ca. 90 Watt) aber auch auf das maximal erzielte Atemminutenvolumen (40 1/min) und das endinspiratorische Lungenvolumen hatte die Sauerstoffgabe keinen Einfluss. Die Autoren betonen, dass der physiologische Mechanismus, der

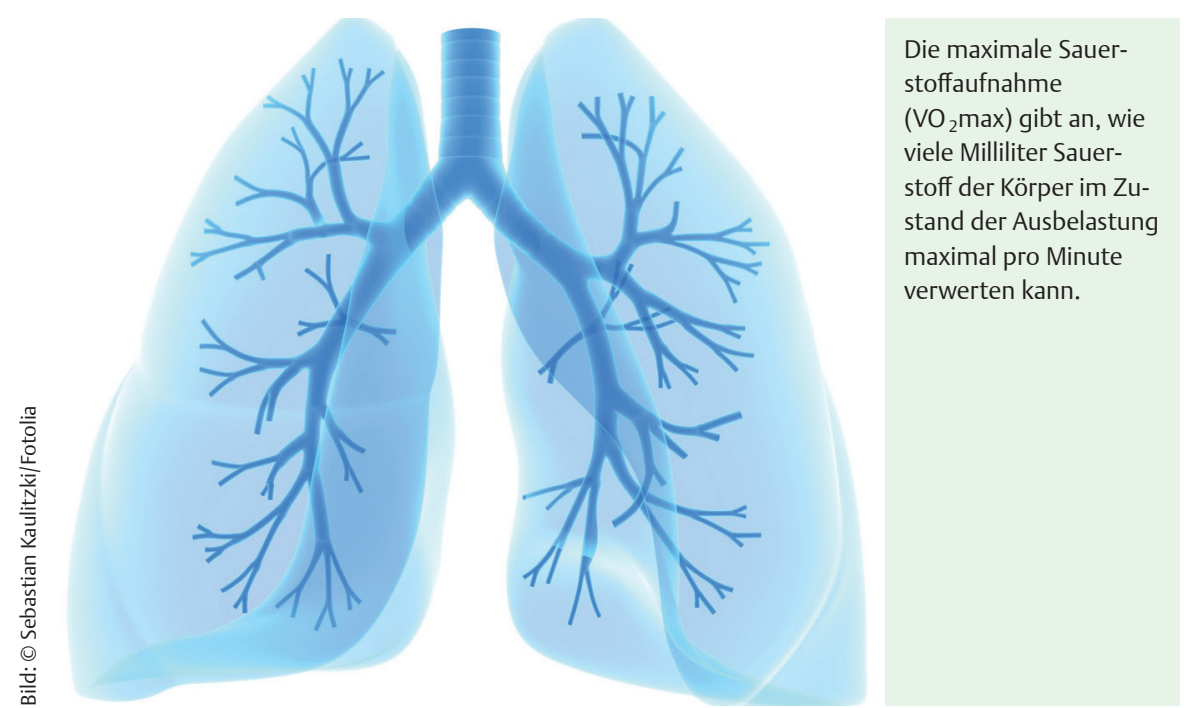

durch die zusätzliche Sauerstoffgabe eine Erhöhung der Sauerstoffaufnahme induziert bisher ungeklärt ist.

\section{Fazit}

Bei der Risikostratifizierung vor einer Lungenteilresektion bei COPD sollte der körperliche Belastungstest modifiziert werden, so die Autoren. Eine geringe zusätzliche Sauerstoffgabe unter Belastung hilft die Patienten zu identifizieren, bei denen eine noch akzeptable prognostische Situation besteht.

Dr. Horst Gross, Berlin

Allergie bei Kindern

\section{Internationaler \\ Konsensus veröffentlicht}

Der internationale Konsensus (ICON) zum Asthma bei Kindern wurde erstmals während des Kongresses der Europäischen Akademie für Allergie und Klinische Immunologie (EAACI) 2012 in Genf präsentiert. Das Ziel des Konsensus ist, die Gemeinsamkeiten der bestehenden Richtlinien $\mathrm{zu}$ ordnen und zusammenzufassen. ICON definiert Empfehlungen für den bestmöglichen Umgang mit der Erkrankung.

„Trotz wirksamer Behandlungsmethoden haben nur weniger als die Hälfte aller asthmatischen Kinder die Möglichkeit, die Symptome ihrer Krankheit wirksam zu bekämpfen", so Prof. Nikos Papadopoulos, EAACI-Generalsekretär und PediatricAsthma-ICON-Chair. Er sagt weiter: „Mit dem Konsensus wollen wir deutlich machen, dass Asthma bei Kindern wirksam behandelt werden kann, vorausgesetzt, Patient und Arzt halten sich an einen individuell vereinbarten Behandlungsplan. Regelmäßige Untersuchungen sind nötig, um die Wirksamkeit der Maßnahmen zu erhöhen und die Behandlung so weit wie möglich an die Bedürfnisse des Patienten anzupassen.“

Der Kinder-Asthma-Konsensus (Pediatric Asthma ICON) ist Teil der iCAALL-Initiative (International Collaboration in Asthma, Allergy and Immunology) und wird vom Europäischen Journal für Allergie und Klinische Immunologie „Allergy” veröffentlicht.

Mitteilung der EAACI, Zürich 